\title{
Diversité des champignons basidiomycètes à carpophores inféodés à certaines espèces des Caesalpiniaceae du Parc National du W du Niger (Afrique de l'Ouest)
}

\author{
Dahiratou IBRAHIM ${ }^{1}$, Oumarou HAMA ${ }^{2 *}$, Pablo Perez DANIËLS ${ }^{3}$, Maman M. INOUSSA ${ }^{4}$, Moussa \\ BARAGÉ 5 , Toudou ADAM 5 , Maria R. ALCANTARA ${ }^{3}$ \& Félix INFANTÉ 3 \\ 1. Département des Sciences de la Vie et de la Terre, École Normale Supérieure, Université Abdou Moumouni de \\ Niamey, BP. 10963, Niamey (NIGER). \\ 2. Département des Productions Végétales et de l'Irrigation, Faculté des Sciences Agronomiques, Université de Tahoua, \\ BP. 255, Tahoua (NIGER). Email : oumahama@gmail.com \\ 3. Dpto. de Botánica, Ecología y Fisiología Vegetal, Ed. Celestino Mutis, $3^{\text {a }}$ pta., Campus de Rabanales. Universidad de \\ Córdoba. 14071, Córdoba (España) \\ 4. Laboratoire Garba Mounkaila, Département de Biologie, Faculté des Sciences et Techniques, Université Abdou \\ Moumouni de Niamey, BP. 10963, Niamey (NIGER) \\ 5. Laboratoire des Productions Végétales, Faculté d'Agronomie, Université Abdou Moumouni, BP. 10960, Niamey \\ (NIGER) \\ "Correspondance : oumahama@gmail.com / hamaoumarou2001@yahoo.fr
}

Original submitted in on $7^{\text {th }}$ June 2017. Published online at www.m.elewa.org on $31^{\text {st }}$ August 2017 http://dx.doi.org/10.4314/jab.v116i1.5

\section{RÉSUMÉ}

Objectif : Ce travail a pour objectif de contribuer à la connaissance des macromycètes du Niger.

Méthodologies et résultats : L'étude a été conduite dans le Parc National du W du Niger qui se trouve dans la zone la plus riche en biodiversité du pays. Ainsi, un inventaire des champignons a été réalisé dans quatre types de formations végétales. II s'agit de la savane arborée plus ou moins arbustive à Afzelia africana et Combretum glutinosum (Site 1) ; de la forêt claire à Isoberlinia doka et Anogeissus leiocarpa (Site 2) ; de la forêt claire à Isoberlinia doka et Berlinia grandiflora (Site 3) et la savane arborée plus ou moins arbustive à Burkea africana et Terminalia avicennioides (Site 4). Au total, cinquante-six (56) espèces de macromycètes ont été recensées sur les sites explorés. Les espèces ectomycorhiziennes s'élèvent à vingt-quatre (24) dont dix-sept (17) dans le site 1, onze (11) au niveau du site 3 , trois (3) dans le site 2 et une (1) sur le site 4 . Ceci est lié à la présence des certaines essences forestières de la famille des Caesalpiniaceae, à savoir $A$. africana, $I$. doka et $B$. grandiflora.

Conclusion et application des résultats : cinq (5) espèces fongiques ectomycorhiziennes (Amanita odorata, A. subviscosa, Lactarius gymnocarpoides, Phylloporus purpureus et Russula albofloccosa) sont communes à la fois à la savane arborée plus ou moins arbustive à $A$. africana et $C$. micranthum et à la forêt claire à $I$. doka et B. grandiflora.

Mots-clefs : Macromycètes, Basidiomycètes, Écologie, Caesalpiniaceae, Parc National du W, Niger, Afrique de l'Ouest. 
Ibrahim et al., J. Appl. Biosci. 2017 Diversité des champignons basidiomycètes à carpophores inféodés à certaines espèces des Caesalpiniaceae du Parc National du W du Niger (Afrique de l'Ouest)

Diversity of carpophoric basidiomycete fungi infected with some species of Caesalpiniaceae from W Niger National Park (West Africa)

\begin{abstract}
Objective: to contribute to the better understanding of macromycetes of Niger. It was conducted in the W National Park, which is located in the richest area in terms of biodiversity in the country.

Methodologies and results: An inventory of fungi was conducted in four vegetation types: tree and shrub savanna with Afzelia africana and Combretum glutinosum (site 1); woodland with Isoberlinia doka and Anogeissus leiocarpa (Site 2); woodland with Isoberlinia doka and Berlinia grandiflora (site 3) and tree and shrub savanna with Burkea africana and Terminalia avicennioides (Site 4). In total, 56 species of macromycetes were identified at the study area belonging to 22 genera and 21 families. The ectomycorrhizal species amounted to 21 , including 17 in site $1 ; 9$ species in site $3 ; 3$ in site 2 and one in site 4 . This is due to the presence of some tree species from the Caesalpiniaceae family, namely Afzelia africana, Isoberlinia doka, Berlinia grandiflora and Burkea africana.

Conclusion and application of results: Five ectomycorrhizal species (Amanita odorata, A. subviscosa, Lactarius gymnocarpoides, Russula albofloccosa and Phylloporus purpureus) were common tree and shrub savanna with $A$. africana and C. micranthum and woodland with I. doka and B. grandiflora.
\end{abstract}

Keywords: Macrofungy, Basidiomycetes, Ecology, Caesalpiniaceae, W National Park Niger, West Africa.

\section{INTRODUCTION}

Les champignons jouent un rôle essentiel dans le fonctionnement des écosystèmes (Moreau et al., 2002). Plusieurs travaux ont montré qu'une grande diversité de champignons symbiotiques sont associés à plusieurs espèces de plantes (Wang et Qiu, 2006 ; Rinaldi et al., 2008). Cependant, des études faites au Sénégal (Thöen et Bâ, 1989), au Burkina Faso (Sanon et al., 1997 ; Guissou et al., 2005), au Nigéria (Redhead, 1968) et en Côte d'Ivoire (Rambelli, 1973) ont montré un faible nombre d'espèces ligneuses à ectomycorhizes. Par ailleurs, en Afrique de l'Ouest, beaucoup des recherches sur les champignons supérieurs ont été effectuées dans les forêts et réserves soudaniennes du Sénégal, de la Guinée, du Bénin et du Burkina Faso (De Kesel et al., 2002 ; Guissou et al., 2005 ; Rivière et al., 2007 ; Sanon et al., 2009 ; Diédhiou et al., 2010 ; Bâ et al., 2011). II ressort que les arbres forestiers à ectomycorhizes appartiennent aux

\section{MATERIEL ET METHODES}

Zone d'étude: L'étude a été réalisée dans le Parc National du W du Niger situé en Afrique de l'Ouest entre $11^{\circ} 50^{\prime}$ et $12^{\circ} 35^{\prime}$ de latitude Nord et $2^{\circ}$ et $2^{\circ} 50^{\prime}$ de longitude Est (figure 1). II couvre une superficie de 220000 ha, et fait partie d'un ensemble d'aires protégées du complexe écologique WAP (W, Arley, Pendjari), reparti familles des Caesalpiniaceae, Dipterocarpaceae et Euphorbiaceae. Dans le Parc National du W du Niger, de nombreux travaux ont porté sur la biodiversité animale et végétale (Mahamane, 2005 ; Audrey, 2007 ; Hibert, 2007 ; Diouf et al., 2010), mais il manque des données sur la diversité des macromycètes de cette aire protégée de la catégorie II de l'UICN. Cette lacune dans la prise en compte des champignons dans les programmes de gestion durable des ressources naturelles est une erreur monumentale vue l'importance de la composante dans la biodiversité, la dynamique des écosystèmes et l'intérêt des espèces comestibles (Hawkswarth, 1991).L'objectif général de cet article est de contribuer à la connaissance champignons supérieurs, ainsi que de leur écologie dans le Parc National du W. Plus spécifiquement, il s'agit (i) d'étudier la diversité spécifique des champignons supérieurs et (ii) d'évaluer leur valeur bio-indicateur.

entre le Bénin, le Burkina Faso et le Niger. Ce Parc est situé au Sud de la commune rurale de Tamou, dans le département de Say. La particularité de la réserve est qu'elle renferme en elle seule, plus de $80 \%$ de la diversité biologique du Niger. Elle est limitée au Nord par la rivière Tapoa, à l'Est par le fleuve Niger, au Sud par la rivière 

certaines espèces des Caesalpiniaceae du Parc National du W du Niger (Afrique de l'Ouest)

Mékrou (Bénin) et à l'Ouest par le Parc d'Arly (Burkina Faso). Le climat est caractérisé par des pluviométries relativement bonnes par rapport aux autres zones du pays. Elle fluctue entre 500 et $800 \mathrm{~mm}$ avec une moyenne annuelle de $700 \mathrm{~mm}$ (figure 2). Les collectes ont été réalisées dans quatre types de formations végétales, à savoir :

Le site 1: Haousa ( $\left.: 12^{\circ} 14^{\prime} 49^{\prime \prime}-\mathrm{E}: 02^{\circ} 22^{\prime} 32^{\prime \prime}\right)$ est une savane arborée plus ou moins arbustive à Afzelia africana et Combretum glutinosum;
Le site 2 : GSKI ( $\left.\mathrm{N}: 12^{\circ} 15^{\prime} 17^{\prime \prime}-\mathrm{E}: 02^{\circ} 23^{\prime} 21^{\prime \prime}\right)$, est une forêt claire à Isoberlinia doka et Anogeissus leiocarpa ;

Le site 3 : GSK II (N : $\left.12^{\circ} 26^{\prime} 51^{\prime \prime}-\mathrm{E}: 02^{\circ} 25^{\prime} 07^{\prime \prime}\right)$ est une forêt claire à Isoberlinia doka et Berlinia grandifora ;

Le site 4 : GSK III (N : $12^{\circ} 28^{\prime} 13^{\prime \prime}$ - E : $\left.02^{\circ} 28^{\prime} 00^{\prime \prime}\right)$, c'est une savane arborée plus ou moins arbustive à Burkea africana et Terminalia avicennioides.

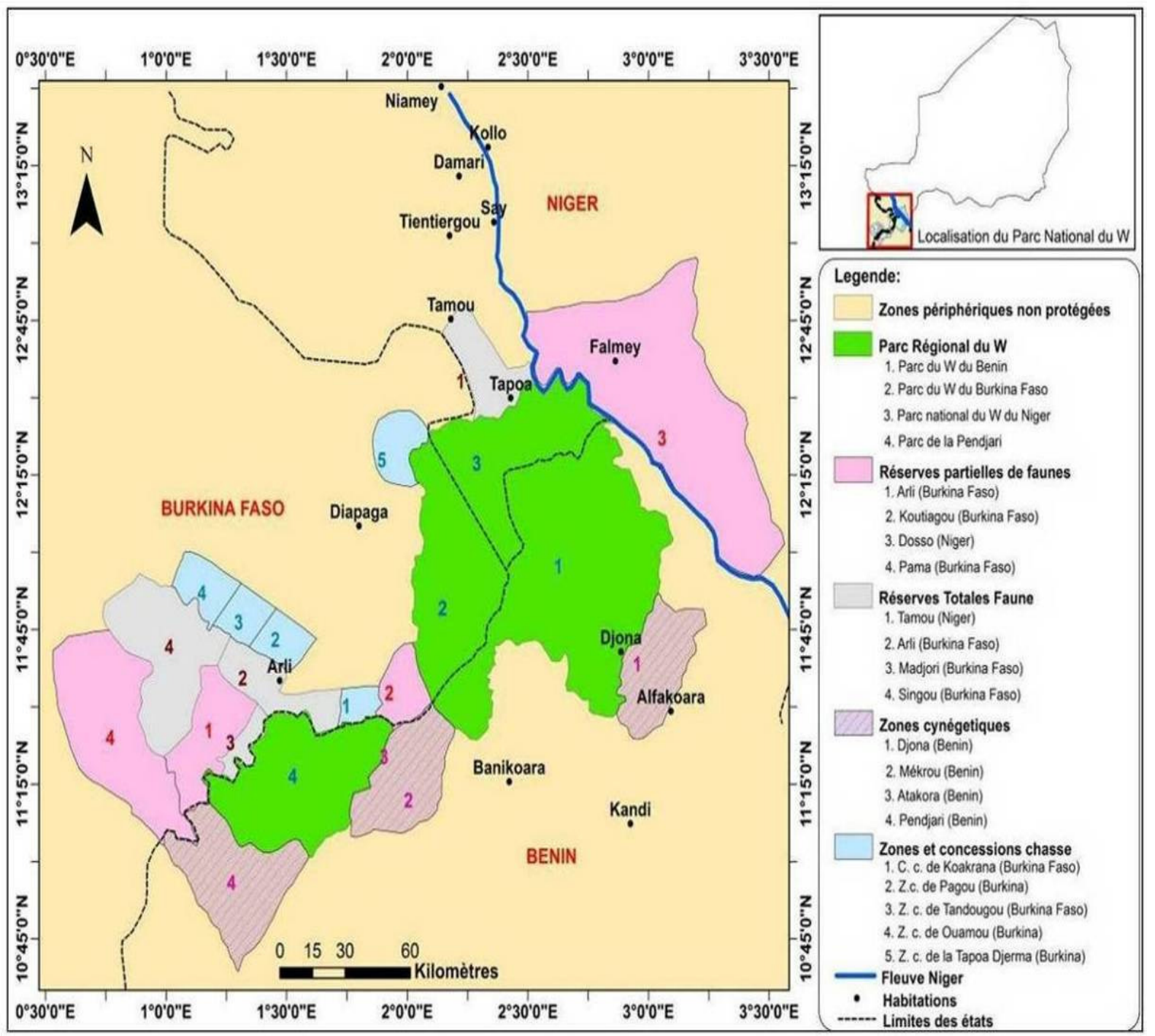

Figure 1. Localisation du Parc National du W du Niger (3) dans le Parc Régional du W en Afrique de l'Ouest 


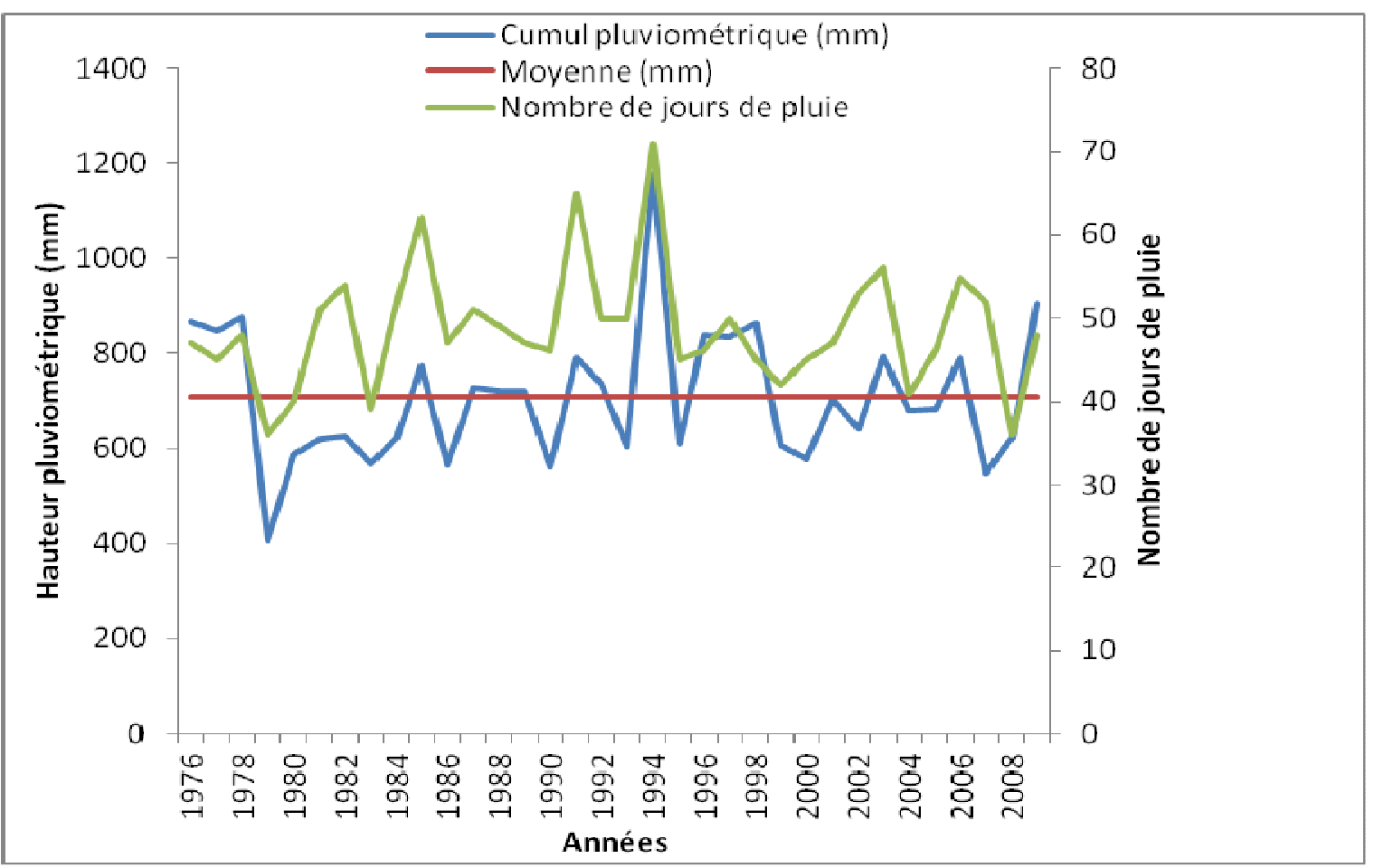

Figure 2. Evolution des précipitations à la station de la Tapoa, au Parc National du W du Niger de 1976-2009.

Collecte et identifications des données : La collecte des données s'est déroulée au cours des périodes de juillet-septembre 2008 à 2012 (Hama, 2012). Ainsi, après avoir identifié les sites, des relevés périodiques ont été effectués pendant les saisons des pluies. Pour ce faire, les carpophores de basidiomycètes ont été photographiés, avant d'être recensés exhaustivement. Aussi, les spécimens ont été séchés et mis en herbier selon la méthode proposée par De Kesel et al. (2002). Les spécimens sont déposés dans l'herbier de l'Université Abdou Moumouni de Niamey (UAM, Niger) et les duplicata sont déposés dans l'herbier de la Faculté des Sciences de l'Université de Cordoba (COFC-F, Espagne) et du Jardin Botanique National, Meise (BR, Bruxelles). Les contrôles des identifications ont été faits à l'aide des travaux suivants : la flore Iconographique des champignons du Congo (Heinemann, 1954; 1956;

\section{RÉSULTATS}

Classification des espèces: Parmi les espèces collectées, on note la présence de vingt-quatre (24) espèces ectomycorhiziennes, réparties dans sept familles, à savoir les Amanitaceae (7 espèces), Russulaceae (10 espèces), Boletaceae (2 espèces), Clavulinaceae (2 espèce), Sclerodermaceae (1 espèce), Hymenochaetaceae (1 espèce) et Xerocomaceae (1 espèce) (figure 3 ). Ces espèces sont associées à
1957); la flore illustrée des champignons d'Afrique Centrale (Heinemann, 1975; 1978; Heinemann et Rammeloo, 1989) et la flore d'Agarics d'Afrique de l'Est (Pegler, 1977) et Härkönen et al. (2003).

Diversité mycofloristique: La classification des champignons récoltés sur les quatre sites est faite en fonction de leur rôle écologique. Ils peuvent être ectomycorhiziens, donc étroitement liés à certaines essences forestières. Ainsi, au cours de la symbiose, le partenaire fongique induit des modifications morphologiques des racines secondaires de l'hôte pour former des ectomycorhizes, qui prospectent le sol au plus large volume (Diop et al., 2004). Aussi, les saprophytes contribuent à la transformation de la litière brute en humus en décomposant la matière organique produite dans cet écosystème.

certaines espèces de Caesalpiniaceae, à savoir Afzelia africana, Isoberlinia doka et Berlinia grandiflora. Aussi, cinq espèces ectomycorhiziennes (Amanita odorata, $A$. subviscosa, Lactarius gymnocarpoides, Phylloporus purpureus et Russula albofloccosa) sont à la fois communes à la savane arborée plus ou moins arbustive à Afzelia africana et Combretum glutinosum et à la forêt claire à Isoberlinia doka et Berlinia grandiflora. Vingt-cinq 

certaines espèces des Caesalpiniaceae du Parc National du W du Niger (Afrique de l'Ouest)

(25) espèces saprotrophes inféodées aux milieux riches en matière organique ont été inventoriées. Ces espèces sont réparties dans neuf familles, qui sont les Agaricaceae (11 espèces), Polyporaceae (5 espèces), Podaxaceae (2 espèces), Entolomataceae (1 espèce), Meruliaceae (1 espèce), Pluteaceae (1 espèce), Xylariaceae (2 espèces), Fomitopsidaceae (1 espèce) et Marasmiaceae (1 espèce). Aussi, six espèces symbiotiques du genre Termitomyces, appartenant à une seule famille (Lyophyllaceae), associées à certaines populations de termites de la famille des Macrotermitidae et trois espèces de parasites appartenant aux familles des Ganodermataceae (2 espèces) et Hymenochaetaceae (1 espèce) ont aussi été inventoriées.

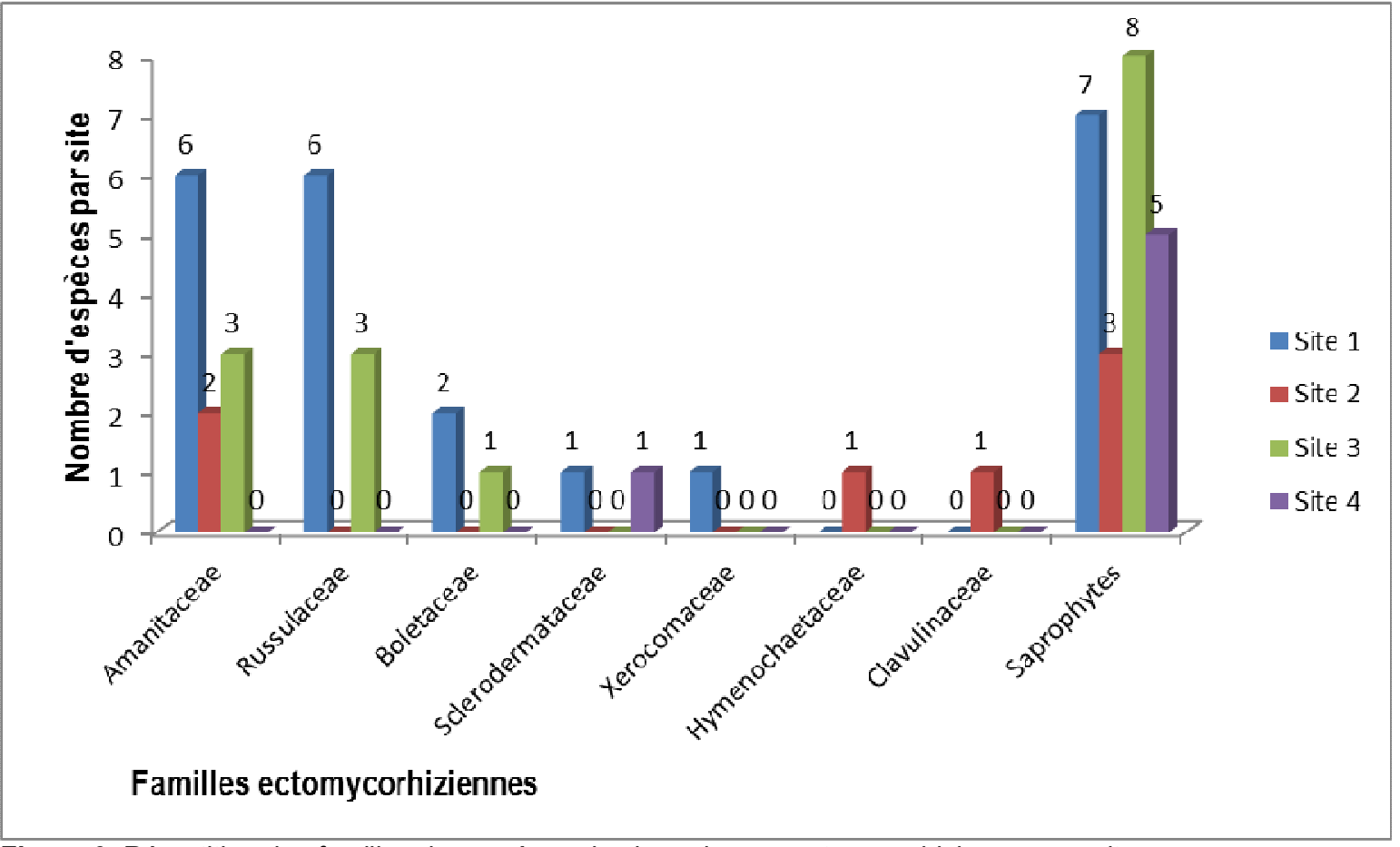

Figure 3. Répartition des familles des espèces de champignons ectomycorhiziennes par site.

Diversité éco-spécifique : Au niveau du site 1 (Savane arborée +/- arbustive à Afzelia africana et Combretum micranthum), trente-cinq (35) espèces de macromycètes réparties dans douze (12) genres et dix (10) familles ont été inventoriées. Dans ces espèces, soixante pour cent $(60 \%)$ font partie du groupe des ectomycorhiziens, trentedeux pour cent $(32 \%)$ des saprotrophes et huit pour cent $(8 \%)$ des symbiontes associés aux termites. Dans ce biotope, les symbiontes ectomycorhiziens, sont principalement associées à Afzelia africana ou à Isoberlinia doka. Ces espèces de champignons sont réparties dans les genres Amanita, Lactarius, Phylloporus, Russula, Scleroderma et Xerocomus. La figure 4 donne une illustration sur la répartition des espèces selon les groupes écologiques. En ce qui concerne le site 2 (Forêt claire à Isoberlinia doka et Anogeissus leiocarpa), on note 10 espèces réparties dans sept (7) genres et huit (8) familles (tableau 3). Dans cette formation végétale, il existe quatre groupes de champignons supérieurs, qui sont les espèces saprophytiques plus dominantes ( $40 \%$ ), les symbiontes ectomycorhiziens qui représentent $30 \%$ des espèces inventoriées, les parasites qui représentent $20 \%$ et les espèces symbiotiques associées aux termites qui sont les moins représentées (10\%). 


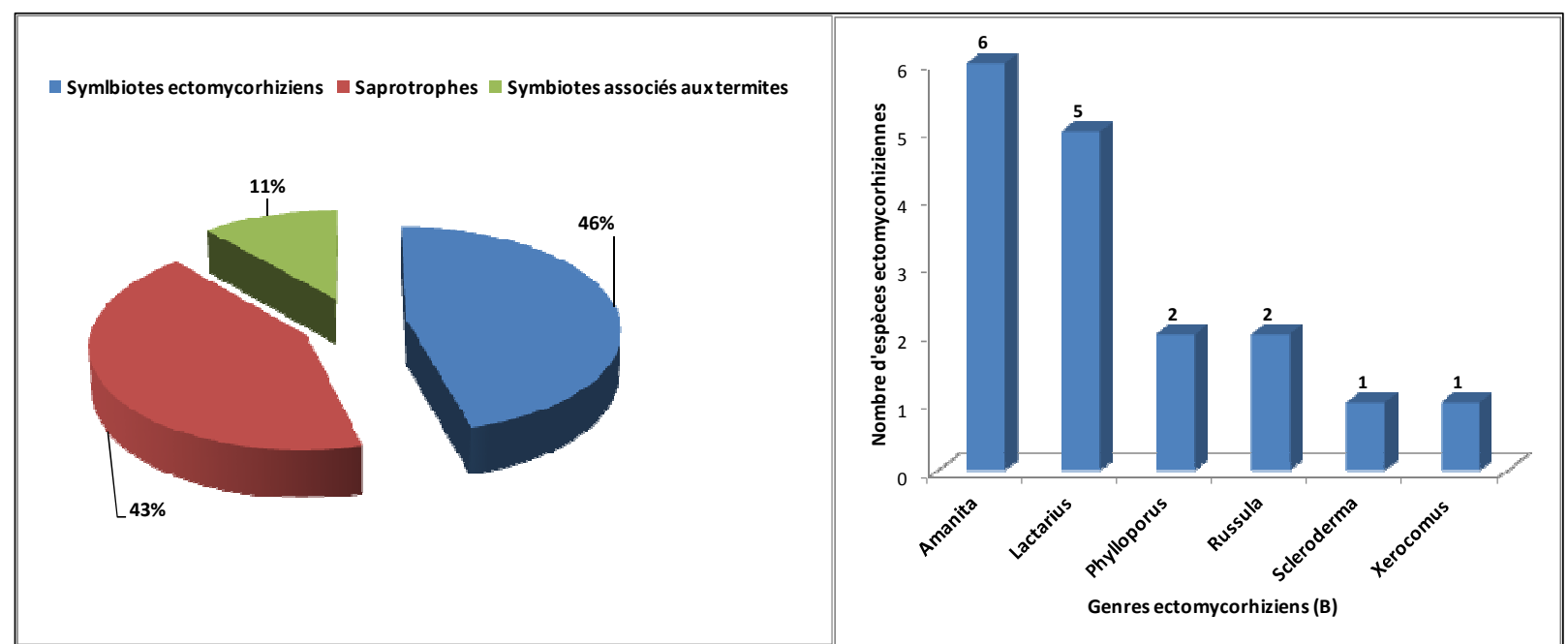

Figure 4. Répartition des espèces par groupes et par genres dans la savane arborée +/- arbustive à Afzelia africana et Combretum micranthum (site 1).

Les saprotrophes sont inféodés aux milieux riches en matière organique, alors que les espèces ectomycorhiziennes sont associées à certaines essences forestières de la famille des Caesalipiniaceae, à savoir Afzelia africana, Isoberlinia doka et Berlinia grandiflora.
Ces espèces ectomycorhiziennes sont exclusivement représentées par deux genres, à savoir Amanita et Scleroderma. La figure 5 donne une illustration quant à la taille et à la répartition des espèces suivant leur groupe fonctionnel dans la forêt claire à l. doka et A.leiocarpa.

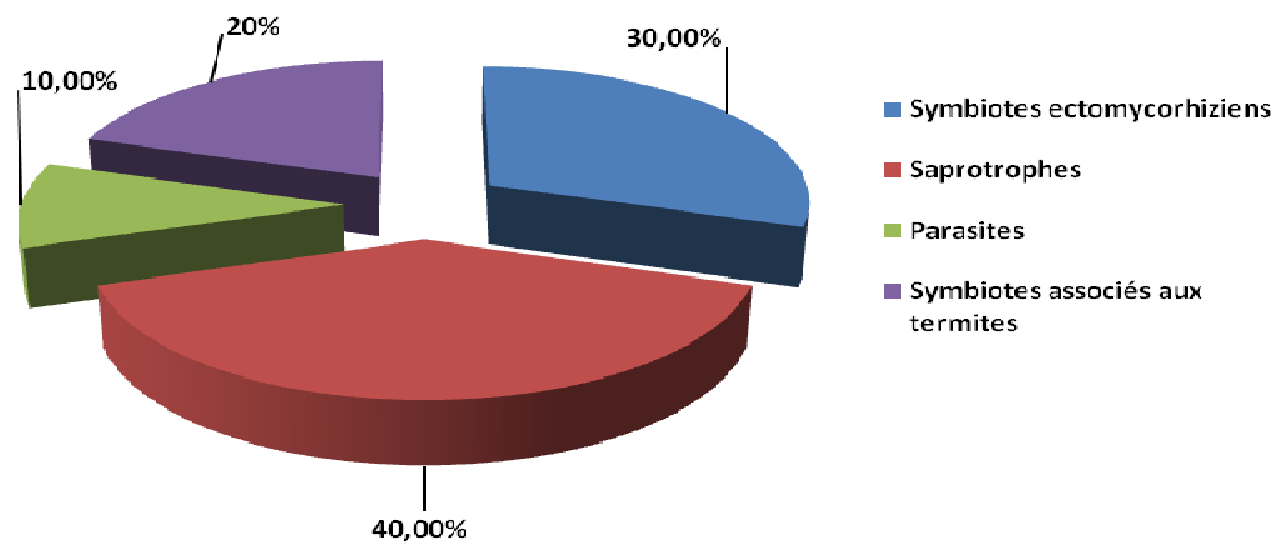

Figure 5. Répartition des espèces par groupe fonctionnel dans la forêt claire à Isoberlinia doka et Anogeissus leiocarpa.

La diversité du site 3 (Forêt claire à Isoberlinia doka et Berlinia grandiflora) est de vingt-une (21) espèces appartenant à quatorze (14) genres qui ont été inventoriées réparties dans dix (10) familles. II convient de noter que la forêt claire à Isoberlinia doka et Berlinia grandiflora présente trois groupes de champignons (figure 6). II s'agit des symbiotes ectomycorhiziens, qui sont les plus dominantes $(52,38 \%)$ et qui totalisent onze (11) espèces avec les saprotrophes qui représentent 23,81\% des espèces, les parasites $(14,29 \%)$ et les symbiotes sont les moins représentées $(9,52 \%)$. Les parasites $(14,29 \%$ des espèces) vivent le plus souvent sur les troncs, ou racines d'espèces ligneuses (Isoberlina doka). La plupart des espèces inventoriées font partie des genres Amanita, Lactarius et Russula (figure 6). 

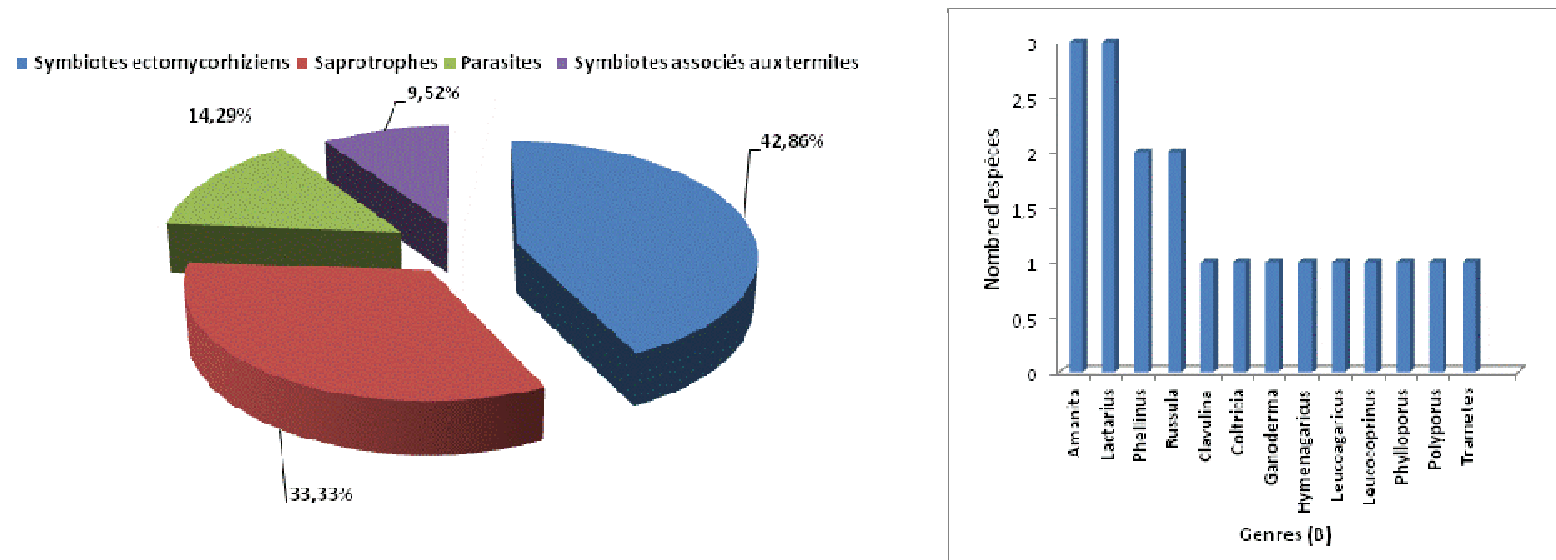

Figure 6. Répartitions des espèces par groupe fonctionnel dans la forêt claire à Isoberlinia doka et Berlinia grandiflora (A) et par genres (B).

En fin le site 4 (Savane arborée plus ou moins arbustive à Burkea africana et Terminalia avicennioides) présente très peu de macromycètes. En effet, une seule espèce ectomycorhizienne (Multiclavula clara) appartenant à la famille des Clavulinaceae a été retrouvée durant la période de prospections mycologiques (2008-2012).

Diversité des espèces ectomycorhiziennes par site de collecte: II a été observé une prédominance des espèces ectomycorhiziennes dans la savane arborée plus ou moins arbustive à Afzelia africana et Combretum glutinosum que les forêts claires à Isoberlinia doka. Ainsi, cette savane présente dix-sept (17) espèces de champignons ectomycorhiziens, dont six (6) amanites (Amanita odorata, A. aff. xanthogala, A. subviscosa, A. masasiensis, A. aff. ovalispora, A. aff. argentea), cinq (5) lactaires (Lactarius edulis, $L$. flammans, $L$. gymnocarpoides, L. pumilus, L. saponaceus), deux (2) espèces de genre Phylloporus ( $P$. ampliporus et $P$. purpureus), deux (2) russules (Russula albofloccosa et $R$. aff. albofloccosa), un (1) scléroderme (Scleroderma dictyosporum) et un (1) Xerocomus (X. linderi). Dans la forêt claire à Isoberlinia doka et Anogeissus leiocarpa, on note la présence de deux (2) espèces ectomycorhiziennes, à savoir Amanita subviscosa et $A$. aff. xanthogala. Ceci est dû au caractère monospécifique en Caesalpiniaceae de ce site. Cependant, onze espèces ectomycorhiziennes sont inféodées à la forêt claire à Isoberlinia doka et Berlinia grandiflora. Elles sont réparties en trois (3) espèces d'amanites (Amanita odorata, A. calopus, A. subviscosa), trois (3) espèces de lactaires (Lactarius aff. emergens, $L$. tenellus, $L$. gymnocarpoides), deux (2) espèces de russules (Russula aff. cinerella et $R$. albofloccosa), une espèce de clavaire (Clavulina ornatipes), une espèce de Coltricia (C. pyrophila) et une espèce de Phylloporus ( $P$. purpureus). Cinq espèces ectomycorhiziennes sont propres à la fois à la savane arborée plus ou moins arbustive à Afzelia africana et Combretum glutinosum et à la forêt claire à Isoberlinia doka. II s'agit de Amanita odorata, A. subviscosa, Lactarius gymnocarpoides, Phylloporus purpureus et Russula albofloccosa (figure 6). 
Ibrahim et al., J. Appl. Biosci. 2017 Diversité des champignons basidiomycètes à carpophores inféodés à certaines espèces des Caesalpiniaceae du Parc National du W du Niger (Afrique de l'Ouest)

Annexe 1 : Liste de quelques espèces ectomycorhiziennes photographiées et collectées
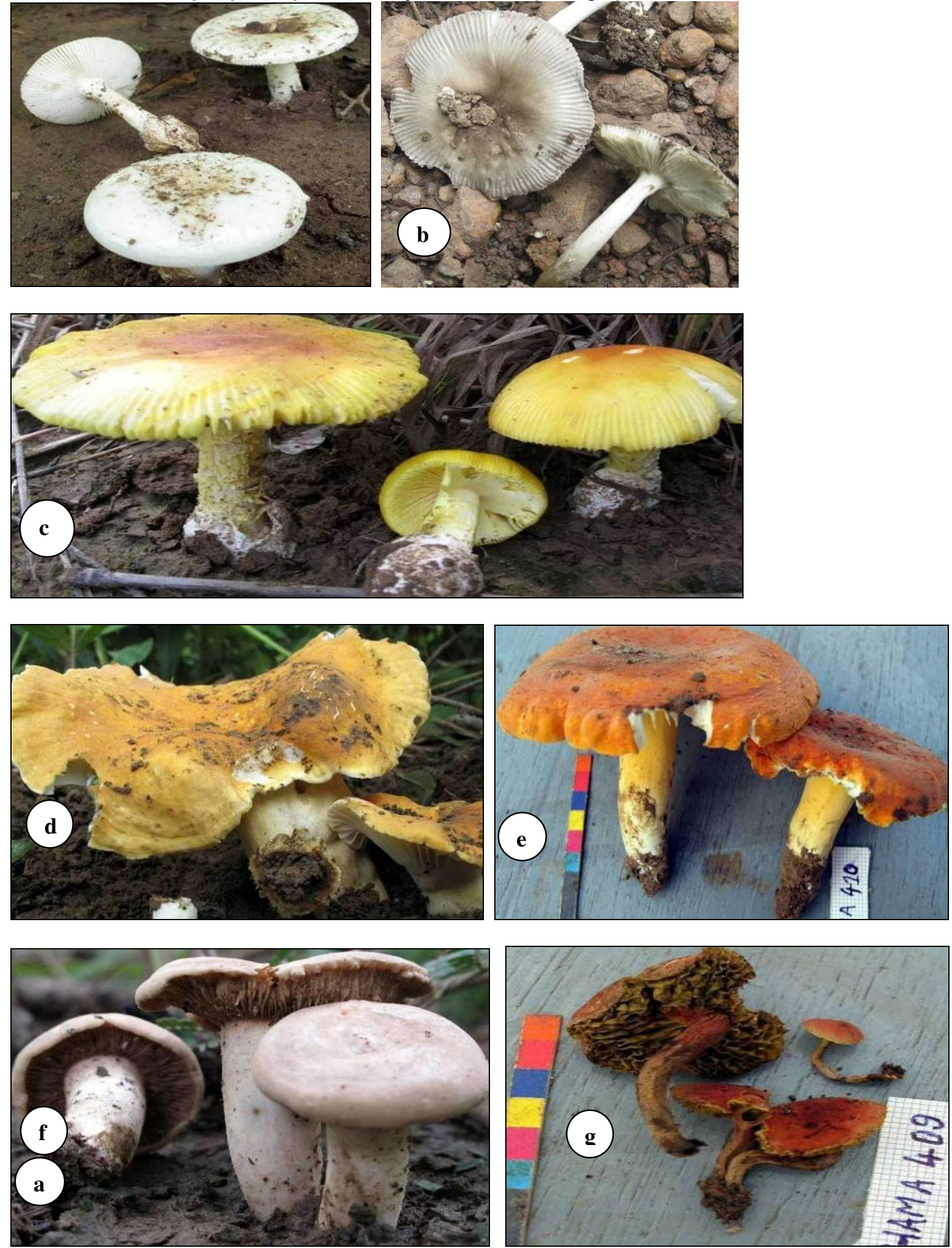

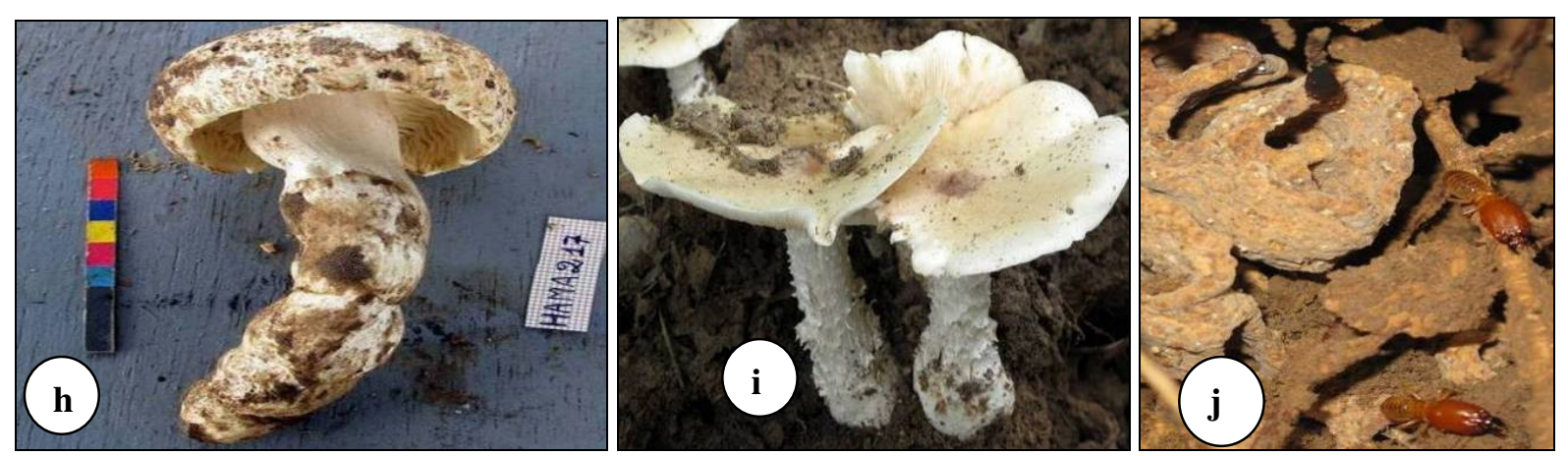

a. Amanita subviscosa Beeli; b. Amanita aff. ovalispora Boedijn; c. Amanita masasiensis Härk. \& Saarim. ; d. Lactarius edulis Verbeken \& Buyck; e. Lactarius flammans Verbeken; f. Lactarius saponaceus Verbeken; g. Phylloporus purpureus, var. ambiguous Heinem; h. Russula albofloccosa Buyck; i. Termitomyces bulborhizus T.Z. Wei, Yao, Wang \& Pegler Pat.; j. Meule contenant Macrotermes subhyalinus associée à T. bulborhizus.

\section{DISCUSSION ET CONCLUSION}

La savane arborée plus ou moins arbustive à Afzelia africana et Combretum glutinosum (site Haoussa) présente plus d'espèces ectomycorhiziennes que la forêt claire à Isoberlinia doka et Berlinia grandiflora (site 3). Ceci se justifie par la présence des vieux pieds d'Isoberlinia doka dans le premier site. Ainsi, les deux essences forestières ( $A$. africana, I. doka) sont considérées comme des plantes hôtes des champignons ectomycorhiziens (Ducousso et al., 1999). En effet, Ducousso et al. (1999) ont décrits 70 espèces de champignons associées principalement à Isoberlinia doka et 34 avec Afzelia africana (Thöen et Bâ, 1989). Ainsi, Lactarius pumilus est une espèce ectomycorhizienne, qui fructifie selon un mode de vie grégaire, parfois cespiteux, rarement isolé, dans la savane arborée à Afzelia africana du Parc National du W. Elle est également présente dans les savanes soudaniennes à Afzelia africana et Isoberlinia doka du Bénin, du Togo et du Sénégal (Maba, 2010 ; Verbeken et Walleyn, 2010) et dans les forêts claires de type miombo du Keynia, de la Tanzanie, de la Zambie et du Zimbabwe (Härkönen et al., 2003). La savane arborée plus moins arbustive à Burkea africana et Terminalia avicennioides s'est révélée très pauvre en mycoflore, même si certains auteurs ont cité Burkea africana parmi les Caesalpiniaceae réputées pour former des relations symbiotiques avec certaines espèces de champignons (Yorou et al., 2002). Cependant, dans les successions primaires, comme dans les successions secondaires, les communautés de champignons ectomycorhiziens se succèdent dans le temps et dans l'espace selon l'âge des formations végétales (Lilleskov et Bruns, 2003). Ainsi, plusieurs travaux suggèrent que les symbiotes ectomycorhiziens s'installent en fonction de l'âge des arbres selon un processus de succession et/ou d'addition
(Bâ et al., 2011). En ce qui concerne la répartition des champignons ectomycorhiziens sur les sites, il y a lieu de noter que la plupart des lactaires (Lactarius edulis, $L$. flammans, $L$. gymnocarpoides, $L$. pumilus, $L$. saponaceus, $L$. aff. emergens, $L$. tenellus) et amanites (Amanita odorata, A. aff. xanthogala, A. subviscosa, $A$. masasiensis, $A$. aff. ovalispora, A. aff. argentea) sont inféodées à la savane arborée plus ou moins arbustive à Afzelia africana. Ce phénomène est surtout lié à l'importance des deux essences forestières dans cette formation forestière, à savoir Afzelia africana et Isoberlinia doka. Ce groupe de champignons joue un rôle écologique très important en favorisant la croissance des forêts naturelles. Ainsi, leur action bénéfique sur leurs hôtes se situe principalement au niveau de la gestion de l'eau et des éléments minéraux peu mobiles, comme le phosphore, mais aussi une meilleure protection des racines contre d'éventuels pathogènes (Egli et Ayer, 1997 ; Yorou et De Kesel, 2002 ; Guillot et Chaumeton, 2003). Cette stratégie reste le principal mode de survie des arbres en Afrique tropicale sèche caractérisée par des sols pauvres en azote et phosphore (Béreau et al., 2003 ; Yorou et De Kesel, 2011). La destruction de ces forêts naturelles entraînerait toujours la mort des mycéliums associés et par conséquent la disparition de dizaines d'espèces de champignons communes et rares (De Kesel et Guelly, 2007). Bien que les russules soient le groupe de badisidiomycètes le plus représenté en Afrique intertropicale (Buyck, 1994), ce genre est moins représenté dans les sites d'étude. Russula albofloccosa est une espèce présente dans la savane arborée plus ou moins arbustive à Afzelia africana et la forêt claire à Isoberlinia doka du Parc National du W du Niger. Elle est également présente dans les forêts claires à Afzelia 

certaines espèces des Caesalpiniaceae du Parc National du W du Niger (Afrique de l'Ouest)

africana de la province de Koloba au Sénégal et dans les prairies du haut Katanga, au Zaïre (Buyck, 1997). La rareté d'espèces fongiques recensées au niveau du site à Burkea africana et Terminalia avicennioides s'explique par la nature sableuse, ainsi que la pauvreté du sol, et son emplacement sur le bassin versant entrainant des eaux de ruissellement. En effet, ce site présente l'aspect de vieux champs dégradés. Cela est dû à la pauvreté des sols, reflétant un ancien champ dégradé et abandonné, marqué par la présence des plages encroutées auxquelles viennent s'ajouter l'abondance des espèces herbacées indicatrices des sols pauvres, telles que Eragrostis tremula et Ceratotheca sesamoides. L'impact du facteur sol reste primordial en régulant fortement la composition spécifique des communautés fongiques.

Aussi, il convient de noter la présence de six espèces de Termitomyces (Termitomyces striatus f. annulatus, $T$. striatus s.l., $T$. striatus, $T$. striatus f. griseiumboides, $T$. microcarpus, $T$. bulborhizus) dans les trois types de formations forestières, à savoir la savane arborée plus ou moins arbustive et les deux types de forêts claires du Parc National du W. Ceci est lié à la présence des termitières épigées où l'on retrouve certaines populations de termites de la famille des Macrotermitidae à savoir Macrotermes subhyalinus, Pseudocanthotermes militaris

\section{REMERCIEMENTS}

Les auteurs remercient l'Université Abdou Moumouni de Niamey (Niger), I'Université de Cordoba (Espagne), l'Agence Espagnole de Coopération Internationale pour le

\section{REFERENCES BIBLIOGRAPHIQUES}

Audrey I, 2007. Caractéristiques et déterminants de la distribution spatio-temporelle de la population d'éléphants du Parc Régional du W, Afrique de l'Ouest. Thèse de Doctorat, Ecole Nationale Supérieure Agronomique de Rennes 222p + annexes.

Bâ A, Duponnois R, Diabaté M, Dreyfus B, 2011. Les champignons ectomycorhiziens des arbres forestiers en Afrique de l'Ouest. Méthodes d'étude, diversité, écologie, utilisation en foresterie et comestibilité. IRD éditions, Marseille, 268 pages

Bâ AM, 1986. Premiers résultats sur l'infection ectomycorhizienne chez deux essences forestières du sud du Sénégal : Afzelia africana Sm. et Uapaca guineensis Mull. Arg. In : Les arbres fixateurs d'azote et l'amélioration biologique de la fertilité du sol, CRDI/NFTA/IFS/Orstom, Dakar 17-25 mars : 243-255. et $P$. spiniger (Koné et al., 2011). Cependant, d'autres formations d'espèces de Termitomyces plus nombreuses ont été signalées dans beaucoup de forêts et réserves naturelles d'Afrique tropicale (Eyi Ndong et al., 2011; Koné et al., 2011). Cependant, de nombreuses termitières sont visibles sur les sites. La zone d'étude étant une aire protégée de catégorie II de l'IUCN (2002), on ne peut émettre l'hypothèse de surexploitation des produits non ligneux tels que les termites, ainsi d'autres espèces fauniques associées entrainant la destruction de I'habitat. On peut donc émettre l'hypothèse selon laquelle que parmi les facteurs destructeurs des termitières figurent en bonne place les feux de brousse et l'excavation des termitières par les braconniers frauduleux. Ces activités ne favorisent pas le bon fonctionnement des termitières et la conséquence sur le plan mycologique est la raréfaction des Termitomyces. Aussi, les feux représentent le principal facteur perturbant les forêts d'Afrique tropicale (Bâ et al., 2011). Ainsi, le passage du feu se traduit par une augmentation du $\mathrm{pH}$ et de la disponibilité en carbone, azote et phosphore du sol. De ce fait, seuls les champignons ectomycorhiziens tolérant de fortes teneurs en nutriments pourraient recoloniser les milieux (Jones et al., 2003).

Développement, ainsi que la Coopération Belge pour le soutien matériel et financier, qui a permis la réalisation de cette étude.

Bâ AM, Buck B, Deschères $P$, Eyssartier $G$, Folno FG, Ducousso M, Wey J, Giraud E, Fontana A, Diallo MAK, Dreyfus B, 2000. Diversity and use of ectomycorrhizal fungi in Guinea tropical rain forest. In GARBAYE J., Lugtenberg B., Sörensen J. (eds.): Selection strategies for plant-beneficial microorganisms, Nancy, 3-5 avril.

Bâ AM, Garbaye J and Dexheimer J, 1991. Influence of fungal propagules during the early stage of the time sequence of ectomycorrhizal colonization on Afzelia africana Sm. seedlings. Canadian Journal of Botany, 66 : 2442-2447.

Baxter JW and Dighton J, 2005. Diversity-functioning relationships in ectomycorrhizal fungal communities. In: Dighton J., White J.F., and Oudemans P. (Eds.) The fungal community: Its organization and role in the ecosystem, Marcel Dekker, New York, pp. 383398. 
Ibrahim et al., J. Appl. Biosci. 2017 Diversité des champignons basidiomycètes à carpophores inféodés à certaines espèces des Caesalpiniaceae du Parc National du W du Niger (Afrique de l'Ouest)

Béreau M, Louisanna E, Grandcourt A, Garbaye J, 2003. Symbiose mycorhizienne et nutrition minérale. Description et dynamique des milieux forestiers. Rev. For. Fr. LV- numéro spécial : 74-83.

Buyck B, 1994. Ubwoba: Les champignons comestibles de l'Ouest du Burundi. Brussels, Administration Générale de la Coopération au Développement: 123 p.

Buyck B, 1997. Flore illustrée des champignons d'Afrique Centrale, 17 ${ }^{\text {ème }}$ Fascicule, 545-598 + Annexes.

Chilvers G.A, Lapeyrie FF and Horan DP, 1987. Ectomycorrhizal vs endomycorrhizal fungi within the same root system. New Phytologist, 107: 441-448.

De Kesel A, and Guelly AK, 2007. Séminaire de mycologie, Togo (Afrique de l'Ouest) du 2 au 26 juillet 2007, 43 pages.

De Kesel A, Codja JTC and Yorou SN, 2002. Guide des champignons comestibles du Bénin. Cotonou, République du Bénin, Jardin Botanique National de Belgique et Centre International Eco développement Intégré, 275 pages.

Diédhiou AG, Selosse MA, Galiana A, Diabaté M, Dreyfus B, Bâ AM, De Faria, SM, Béna G, 2010. Multi-host ectomycorrhizal fungi are predominant in a Guinean tropical rainforest and shared between canopy trees and seedlings. Environmental Microbiology, 2 (8): 2219-2232.

Diop T, Fall MA, Leye Diagne G, Sy-ndir M, Kaire A, Diallo A, 2004. Atouts et Avantages des champignons pour une agriculture durable. Revue sur l'agriculture durable à faibles apports externes. Les agricultures de la nouvelle génération, 35 pages.

Diouf A, Barbier N, Mahamane A, Lejoly J, Saadou M, Bogaert J, 2010. Caractérisation de la structure spatiale des individus ligneux dans une brousse tachetée au Sud-ouest du Niger. Rev.can. rech. for. $40: 827-835$.

Ducousso M, Louppe D, Ouattara N, Eyssartier G, Buyck $B, 1999$. Des mycorhizes très diversifiées dans les jachères naturelles au nord de la Côte d'Ivoire. In La jachère en Afrique tropicale, rôle, aménagements, alternatives, 13-16.

Egli S, and Ayer F, 1997. Est-il possible d'améliorer la production de champignons comestibles en forêt? L'exemple de la réserve mycologique de la Chanéaz, en Suisse. Rev. For. \& Fr XLIX- $n^{\circ}$ sp.

Eyi Ndong H, Degref J, De Kesel A, 2011. Champignons comestibles des forêts denses d'Afrique centrale Taxonomie et identification abc Taxa vol 10.
Guillot J, Chaumeton H, 2003. Dictionnaire de Champignons et des termes de mycologie, 157 pages.

Guinberteau J, and Courtecuisse R, 1997. Diversité des champignons (surtout mycorhiziens dans les écosystèmes forestiers actuels. Rev. For .Fr. XLIX$\mathrm{n}^{\circ} \mathrm{sp}$.

Guissou KML, Sankara P, Guinko S, 2005. Phlebopus sudanicus ou «viande des Bobos», un champignon comestible dans le département de Satiri au Burkina Faso. Cryptogamie, Mycologie, 26(3) : 195-204.

Hama O, 2012. Diversité et comestibilité des champignons basidiomycètes à carpophores au Sud-ouest du Niger, 210 pages. Thèse de Doctorat Unique, Université Abdou Moumouni de Niamey, Niger

Härkonen M, Niemelä T, Mwasumbi L, 2003. Tanzanian Mushrooms. Edible, harmful and other fungi, Noorlinea 10. Helsinki. Botanical Museum Finnish Museum of National History, University of Helsinki, 200 pages.

Hawkswarth DL, 1991. The fungal dimension of Biodiversity: magnitude, significance and conservation. Mycological Research 95: 641: 655.

Heinemann P, 1954. Boletineae Flore Iconographique des Champignons du Congo 3, 49-80

Heinemann P, 1956. Flore Iconographique des Champignons du Congo, 5 ème Fascicule : Agaricus, 99-119 + Planches.

Heinemann $P, 1975$. Flore illustrée des champignons d'Afrique centrale, Fascicule 4. Volvariella, 75-84 + Planches.

Heinemann $P, 1978$. Flore illustrée des champignons d'Afrique centrale, Fascicule 6. Volvariellacea. Complément. 107-120 + Planches.

Heinemann P and Rammeloo J, 1989. Tubosaeta (Xerocomaceae, Boletineae). Flore illustrée des champignons d'Afrique centrale fasc. 14. Ministère de l'Agriculture-Jardin Botanique National de Meise, Belgique

Hibert F, 2007. Déterminants écologiques et anthropiques de la structuration des peuplements d'ongulés sauvages en Afrique de l'Ouest : cas du Parc Régional du W du fleuve Niger, 207 pages. Thèse de doctorat, Ecole Nationale Supérieure d'Agronomie de Rennes et Université ABDOU Moumouni.

Jones MD, Durall DM, Carney JWG, 2003. Ectomycorrhizal fungal communities in young forest stand regenerating after clearcut logging. New Phytologist, 157: 399-422. 
Ibrahim et al., J. Appl. Biosci. 2017 Diversité des champignons basidiomycètes à carpophores inféodés à certaines espèces des Caesalpiniaceae du Parc National du W du Niger (Afrique de l'Ouest)

Kone NA, Dosso K, Konate S, Kouadio JY, Linsenmair $\mathrm{KE}$, 2011. Environmental and biological determinants of Termitomyces species seasonal fructification in central and southern Côte d'Ivoire. Insect. Soc. 58: 371-382.

Lilleskov EA, Bruns TD, 2003. Root colonization dynamics of two ectomycorrhizal fungi of contrasting life history strategies are mediated by addition of organic nutrient patches. New Phytologist, 159: 141 151.

Locquin M, 1954. Une chanterelle comestible de la Côte d'Ivoire : Hygrophoropsis mangenotii sp. nov. J. Agric. Bot.Trop. Appl., 1 : 359-361.

Maba DL, 2010. Les Lactarius de la réserve de faune d'Alédjo, 56 pages. Mém. DEA en Biologie de Développement, Faculté des Sciences, Université de Lomé (Togo).

Mahamane A, 2005. Étude floristique, phytosociologique et phytogéographique du Parc Régional du W du Niger. Thèse de doctorat en Sciences Agronomiques \& Ingénierie Biologique, Université Libre de Bruxelles (Belgique), 561 pages.

Rambelli A, 1973. The rhizosphere of mycorrhizae. In MARKS G. C., KOZLOWSKI, T.T. (eds.): Ectomycorrhizae: Their Ecology and Physiology, New York, Academic Press: 229-343.

Redhedad JF, 1968. Mycorrhizal associations in some Nigerian forest trees. Transactions of the British Mycological Society, 51: 377-387.

Rinaldi AC, Comandini O, Kuyper TW, 2008. Ectomycorrhizal fungal diversity: Separating the wheat from the chaff. Fungal Divers. $33: 1-45$.

Rivière $T$, Diedhiou $A G$, Diabaté $M$, Senthilarasu $G$, Natarajan K, Verbeken A, Buyck B, Dreyfus B, Bena G, Bâ AM, 2007. Genetic diversity of ectomycorrhizal basidiomycetes from African and Indian tropical forests. Mycorrhiza, 17 : 415-428.

Sanon KB, Bâ AM, Dexheimer J, 1997. Mycorrhizal status of some fungi fruiting beneath indigenous trees in Burkina Faso. Forest Ecology and Management, $98:$ 61-69.

Sanon KB, Bâ AM, Delaruelle C, Duponnois R, Martin F, 2009. Morphological and molecular analyses in Scleroderma species associated with some Caesalpiniaceae, Dipterocarpaceae and Phyllanthaceae trees in southern Burkina Faso. Mycorrhiza, 19: 571-584.

Thöen D and Bä AM, 1989. Ectomycorrhizas and putative ectomycorrhizal fungi of Afzelia africana and Uapaca senegalensis in southern Senegal. New Phytologist, 113:549-559.
Thöen D and Ducousso M, 1989. Champignons et ectomycorhizes du Fouta Djalon. Revue Bois et Forêts des Tropiques, 221, 45-63.

Verbeken A and Walleyn R, 2010. Monograph of Lactarius in Tropical Africa. Fungus Fl. Trop. Afr. 2: $161 \mathrm{pp} .+54 \mathrm{pl}$.

Wang B and Qiu YL, 2006. Phylogenetic distribution and evolution of mycorrhizas in land plants. Mycorrhiza 16: 299-363.

Yorou SN and De Kesel A, 2002. Connaissances ethnomycologiques des peuples Nagot du centre Bénin (Afrique de l'Ouest). In E. ROBBRECHT, J. DE GREEF ETI. FRIIS, eds. Plant Systematics and Phytogeography for the understanding of African biodiversity. Proceedings of the XVith AETFAT Congress 2000, Meise, National Botanic Garden of Belgium. Syst. Geogr. PI. 71: 627-637.

Yorou SN, De Kesel A, Codja JTC, 2002. Diversité et productivité des champignons comestibles de la forêt classée de Wari Maro (Benin, Afrique de l'Ouest). In E. ROBBRECHT, J. DE GREEF \& I. FRIIS, eds. Plant Systematics and Phytogeography for the understanding of African biodiversity. Proceedings of the XVth $A E T F A T$ Congress 2000, Meise, Jardin Botanique National, Belgique. 